Chinese Journal of Organic Chemistry

NOTE

\title{
白藜芦醇、白皮杉醇和赤松素的合成
}

\author{
张静静 $a, b$ 姚 明*,b 李 立 ${ }^{a, b}$ 桑大永 $b$ \\ 熊航行*, $a, b$ 刘生鹏*,a \\ ( ${ }^{a}$ 武汉工程大学化工与制药学院 武汉 430205) \\ ( ${ }^{b}$ 荆楚理工学院化工与药学院 荆门 448000)
}

\begin{abstract}
摘要 研究了白荺芦醇、赤松素和白皮杉醇的一种简便合成方法. 二苯乙烯类化合物在铝和碘的作用下实现双键顺反 异构和芳甲醚甲基的脱除，实现白葱芦醇、赤松素和白皮杉醇的高效合成，反应的总收率分别为 $68 \%, 78 \%$ 和 $56 \%$. 反 应溶剂过滤后可以再次使用. 通过量子化学计算和控制实验认为碘自由基可能是脱甲基过程中导致双键顺反异构的关 键因素.
\end{abstract}

关键词 白藜芦醇; 二苯乙烯; 脱甲基; Wittig 反应; 顺反异构

\section{Synthesis of Resveratrol, Piceatannol and Pinosylvin}

\author{
Zhang, Jingjing ${ }^{a, b} \quad$ Yao, Ming ${ }^{*, b}$ \\ $\mathrm{Li}, \mathrm{Li}^{a, b}$
}

Xiong, Hangxing ${ }^{*, a, b}$

Liu, Shengpeng*,a

$\left({ }^{a}\right.$ School of Chemical Engineering and Pharmacy, Wuhan Institute of Technology, Wuhan 430205)

$\left({ }^{b}\right.$ College of Chemical Engineering and Pharmacy, Jingchu University of Technology, Jingmen 448000)

\begin{abstract}
A convenient method for the practical synthesis of resveratrol, piceatannol and pinosylvin is described. Resveratrol, pinosylvin and piceatannol can be achieved through a simultaneous demethylation and isomerization process from stilbenes with the aid of aluminum and iodine. The overall yields of the reaction were $68 \%, 78 \%$ and $56 \%$ (based on aromatic aldehyde). The solvent of the reaction can be reused after filtered. At the same time, quantum chemical calculations and control experiments show that iodine radical may be the key factor leading to cis-trans isomerization of double bond in the process of demethylation.

Keywords resveratrol; stilbene; demethylation; Wittig reaction; cis-trans isomerisation
\end{abstract}

多羟基二苯乙烯类天然产物具有显著的抗氧化、抗 肿瘤和抗炎等生理活性 ${ }^{[1]}$, 如苯烯莫德可作为治疗过敏 性皮炎、湿疹的药物 ${ }^{[2]}$; 白藜芦醇可作为食品、保健品 和化妆品的重要功能性添加剂 ${ }^{[3]}$. 这类天然产物在在植 物中的含量较低, 从它们的天然来源难以大量获得. 化 学合成方法可以快速大量制备此类天然产物及其类似 物，常见的合成方法有 Perkin 反应、Heck 反应、Wittig 及 Wittig-Horner 反应、Suzuki 偶联反应、Knoevenagel 反应等 ${ }^{[4]}$.

Resveratrol, Piceatannol 和 Pinosylvin 是三种典型的 多羟基二苯乙烯类天然产物, 结构如图 1 所示. 关于这 类天然产物的合成最常用的方法是 Wittig 和 Wittig-
Horner 反应，具体过程是采用甲基保护的醛或酮与磷叶 立德或亚膦酸酯反应形成甲基保护的多羟基二苯乙烯, 再脱掉甲基实现天然产物的合成. 采用 Wittig 或 WittigHorner 反应实现 Resveratrol 的合成, 有较多文献报道 ${ }^{[5]}$, 通过 Wittig 或 Wittig-Horner 反应得到甲基保护的多羟基 二苯乙烯(绝大部分情况下, 该产物含有顺式和反式两 种构型), 再通过碘或者二苯硫醚等促进的异构反应得 到单一反式构型产物, 最后脱掉甲基得到目标产物. 如 Scheme 1 所示, 已知的合成方法在应用中存在一些局 限, 一是需要两步化学反应实现从 Wittig 或者 WittigHorner 反应产物到目标产物的转化; 二是脱甲基常用试 剂为 $\mathrm{AlI}_{3}$ 或者 $\mathrm{BBr}_{3}\left(\mathrm{AlI}_{3}\right.$ 只能现制现用, 不易保存; $\mathrm{BBr}_{3}$

* Corresponding authors. E-mail: yaomingcep@jcut.edu.cn; 731990153@qq.com; liuabss@163.com Received August 9, 2019; revised October 8, 2019; published online December 2, 2019.

Project supported by the Science Technology Foundation for Creative Research Group of Hubei Department of Education (No. T201719). 湖北省高校优秀中青年科技创新团队(No. T201719)资助项目. 
脱甲基反应需要在低温下进行, 反应条件苛刻). 目前 报道比较高效的方法是邹永等报道的可以通过碘化铝 实现脱甲基和双键顺反异构, 但碘化铝需提前制备, 操 作复杂 ${ }^{[7 b]}$. 因此开发更简便更高效地合成多羟基取代 的二苯乙烯类天然产物的方法具有重要的意义.

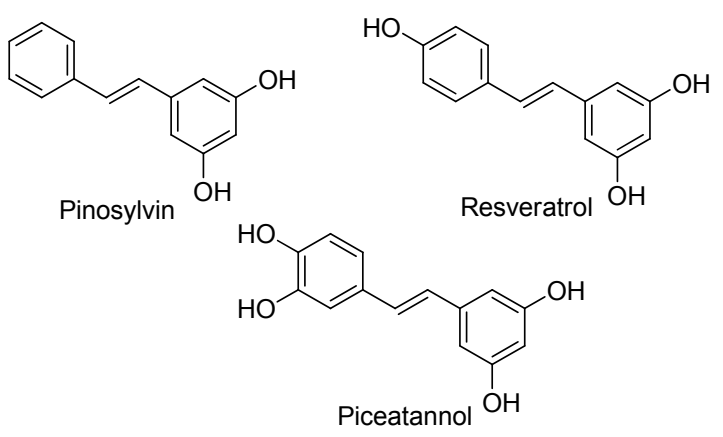

图 1 白藜芦醇、白皮杉醇和赤松素的化学结构

Figure 1 Chemical structures of Resveratrol, Piceatannol and Pinosylvin

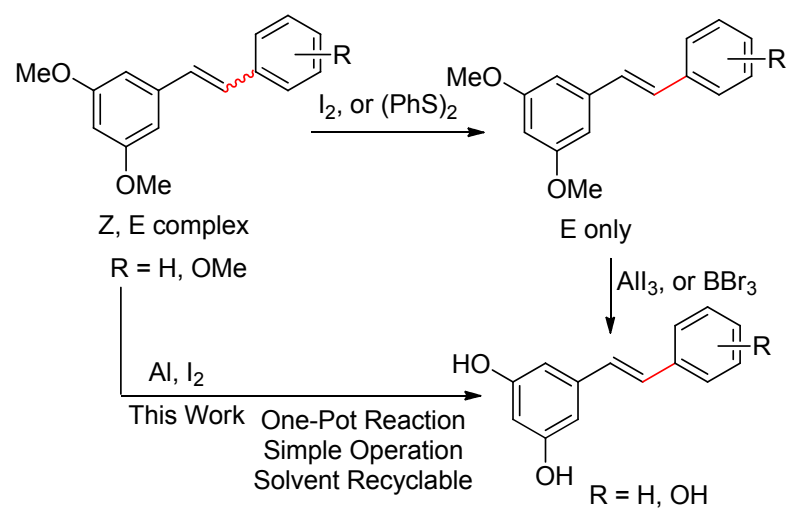

图式 1 脱甲基方法合成反式多羟基取代二苯乙烯类天然产 物

Scheme 1 Synthesis of trans-polyhydroxylated stilbenes via demethylation
在本课题组前期报道的碘化铝脱甲基工作基础 上 $^{[6]}$, 我们进一步报道了以单质铝和单质碘为顺反异构 和脱甲基试剂，一锅反应实现甲基保护的多羟基二苯乙 烯类化合物的顺反异构和脱甲基，绿色高效地实现了 Resveratrol, Piceatannol 和 Pinosylvin 的合成. 在理论计 算和控制实验的基础上，提出了一种更加合理的脱甲基 异构化反应机理.

\section{1 结果与讨论}

白藜芦醇、白皮杉醇和赤松素的合成路线如 Scheme 2 所示. 以 3,5-二甲氧基茮醇为原料, 经三溴化磷溴代 后再与三苯基膦反应形成磷叶立德 $\mathbf{3}$. 化合物 $\mathbf{3}$ 在氢氧 化锂的作用下，与三种不同的醛通过 Wittig 反应形成对 应的甲基保护的多羟基二苯乙烯 $4 \mathbf{a} \sim \mathbf{4 c}$, 为顺反异构 混合物, $Z / E$ 比值大于 1 . 化合物 $\mathbf{4 a} \sim \mathbf{4} \mathbf{c}$ 在铝和碘的作用 下完成甲基的脱除和顺反异构得到白藜芦醇 $5 \mathbf{a}$ 、白皮杉 醇 $5 \mathbf{c}$ 和赤松素 $\mathbf{5 b}$.

\section{1 脱甲基异构化反应优化}

在进行铝和碘脱甲基的过程中，首先进行白㴝芦醇 的合成研究, 得到最优化条件后再进行白皮杉醇和赤松 素的合成, 研究结果如表 1 所示. 脱除化合物 $4 \mathbf{a}$ 的甲基 时, 先制备 $\mathrm{AlI}_{3}$, 再将 $\mathbf{4 a}$ 的乙腈溶液加入并在 $82{ }^{\circ} \mathrm{C}$ 反 应至原料消失，柱层析分离得到的产物为顺反异构混合 物, 顺反比值为 $1: 4$, 与文献 ${ }^{[7 a]}$ 报道的单一反式构型产 物不一致(Entry 1). 将反应溶剂由含水量 $\leqslant 0.01 \%$ 的 HPLC 级乙腈变为文献 ${ }^{[7 b]}$ 使用的市售分析纯乙腈(含水 量 $\leqslant 0.3 \%)$, 反应的收率降低，同时分离得到的产物仍 为顺反异构混合物, 顺反比值为 $1: 8$ (Entry 2). 直接以 碘化铝为作为脱甲基试剂无法直接得到单一反式构型 的目标产物. 我们尝试在含水量 $\leqslant 0.01 \%$ 的 HPLC 级乙

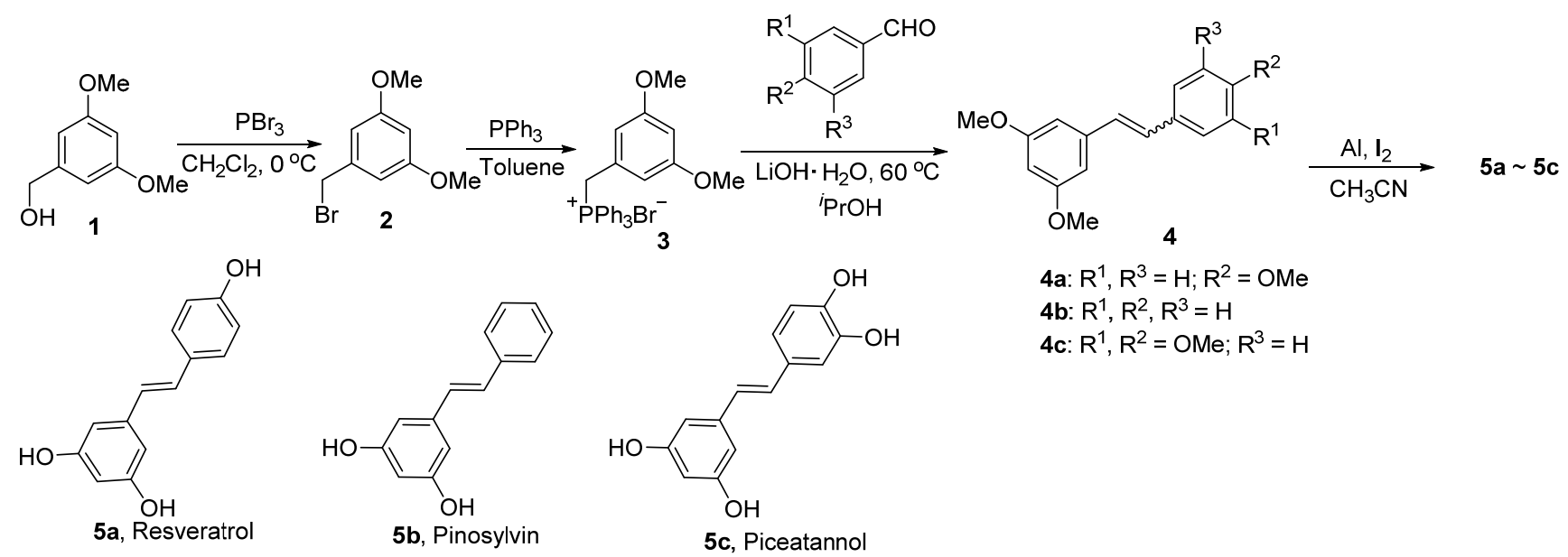

图式 2 白藜芦醇、白皮杉醇和赤松素的合成路线

Scheme 2 Synthetic route toward resveratrol, piceatannol and pinosylvin 
表 1 反应条件优化 ${ }^{a}$

Table 1 Optimization of reaction conditions

\begin{tabular}{|c|c|c|c|c|c|}
\hline Entry & Substrate & Ether-cleaving reagent ${ }^{b}$ & Temperature $/{ }^{\circ} \mathrm{C}$ & Product & Yield/\% \\
\hline 1 & $4 \mathbf{a}$ & $\mathrm{AlI}_{3}(3.3)$ & 82 & $5 \mathbf{a}$ & $65^{c}$ \\
\hline 2 & $4 a$ & $\mathrm{AlI}_{3}(3.3)$ & 82 & $5 \mathbf{a}$ & $62^{d}$ \\
\hline 3 & $4 a$ & $\mathrm{Al}, \mathrm{I}_{2}\left(3.6 \mathrm{Al}, 5.4 \mathrm{I}_{2}\right)$ & 75 & $5 a$ & 81 \\
\hline 4 & $4 \mathbf{a}$ & $\mathrm{Al}, \mathrm{I}_{2}\left(3.6 \mathrm{Al}, 5.4 \mathrm{I}_{2}\right)$ & 75 & $5 \mathbf{a}$ & $66^{g}$ \\
\hline 5 & $4 a$ & $\mathrm{Al}, \mathrm{I}_{2}\left(3.6 \mathrm{Al}, 5.4 \mathrm{I}_{2}\right)$ & 82 & $5 \mathbf{a}$ & 62 \\
\hline 6 & $4 a$ & $\mathrm{Al}, \mathrm{I}_{2}\left(3.9 \mathrm{Al}, 5.85 \mathrm{I}_{2}\right)$ & 75 & $5 \mathbf{a}$ & 82 \\
\hline 7 & $4 a$ & $\mathrm{Al}, \mathrm{I}_{2}\left(3.3 \mathrm{Al}, 4.95 \mathrm{I}_{2}\right)$ & 75 & $5 \mathbf{a}$ & 73 \\
\hline 8 & $4 a$ & $\mathrm{Al}, \mathrm{I}_{2}\left(3.6 \mathrm{Al}, 5.4 \mathrm{I}_{2}\right)$ & 75 & $5 \mathbf{a}$ & $80^{e}$ \\
\hline 9 & $4 \mathbf{a}$ & $\mathrm{Al}, \mathrm{I}_{2}\left(3.6 \mathrm{Al}, 3.6 \mathrm{I}_{2}\right)$ & 75 & $5 \mathbf{a}$ & 42 \\
\hline 10 & $4 a$ & $\mathrm{Al}, \mathrm{I}_{2}\left(3.6 \mathrm{Al}, 7.2 \mathrm{I}_{2}\right)$ & 75 & $5 a$ & $-^{f}$ \\
\hline 11 & $4 a$ & $\mathrm{Al}, \mathrm{I}_{2}\left(7.2 \mathrm{Al}, 3.6 \mathrm{I}_{2}\right)$ & 75 & $5 a$ & 56 \\
\hline 12 & $4 b$ & $\mathrm{Al}, \mathrm{I}_{2}\left(2.4 \mathrm{Al}, 3.6 \mathrm{I}_{2}\right)$ & 75 & $5 \mathbf{b}$ & 87 \\
\hline 13 & $4 c$ & $\mathrm{Al}, \mathrm{I}_{2}\left(4.8 \mathrm{Al}, 7.2 \mathrm{I}_{2}\right)$ & 75 & $5 \mathrm{c}$ & 68 \\
\hline
\end{tabular}

${ }^{a}$ Unless noted otherwise, the acetonitrile used in demethylation is HPLC grade. The water content of the acetonitrile is less than $0.01 \% .{ }^{b}$ Demethylation reagent and its dosage. ${ }^{c}$ The obtained product was a mixture of two isomers in a ratio of $1: 4{ }^{d}$ Acetonitrile is a commercially available analytical reagent with a water content of $\leqslant 0.3 \%$. The obtained product was a mixture of two isomers in a ratio of $1: 8$ (cis-5a/trans-5a). ${ }^{e}$ The yield is the average yield of the two reactions. ${ }^{f}$ After reacted at $75{ }^{\circ} \mathrm{C}$ for $7 \mathrm{~h}$, the reaction could only provide the starting material and unknown polymer. ${ }^{g}$ Acetonitrile is a commercially available analytical reagent with a water content of $\leqslant 0.3 \%$. The obtained product was a single isomer of trans-5a.

腈中将铝、碘(铝和碘的摩尔用量比为 $3.6: 5.4$ )和 $\mathbf{4 a}$ 混 合, 再加热到 $75{ }^{\circ} \mathrm{C}$ 反应 $7 \mathrm{~h}$, 柱层析分离可以到单一反 式构型的化合物 5a, 反应的收率为 81 (Entry 3). 反应的 粗产物进行核磁分析, 未发现顺式构型的产物. 在这一 反应体系中, 铝和碘既作为顺反异构试剂促进双键从顺 式到反式的转变, 又通过二者之间的反应形成 $\mathrm{AlI}_{3}$ 实现 甲基的脱除. 将反应的溶剂调整为市售分析纯乙腈, 反 应的收率明显降低, 但目标产物 $5 \mathbf{a}$ 仍为单一反式构型 产物(Entry 4), 说明溶剂中的水对反应的收率有较大的 影响, 对产物的异构体比例无影响. 将反应的温度升至 $82{ }^{\circ} \mathrm{C}$, 反应的收率明显降低(Entry 5). 在不改变单质铝 和碘的摩尔用量比时, 将脱甲基试剂用量降低, 反应的 收率降低; 脱甲基试剂的用量增高, 反应收率变化不大 (Entries 6,7). 化合物 $\mathbf{4 a}$ 的脱甲基完成后将反应液冷却, 过滤所得滤液作为溶剂参与下一批次的脱甲基反应, 也 可以得到目标目标产物, 两次反应的平均收率可以达到 $80 \%$ (Entry 8), 说明反应的溶剂乙腈可以通过简单的过 滤后重复使用. 改变脱甲基试剂中单质铝和碘的摩尔用 量比例, 反应的收率降低(Entries 9, 11), 特别是当增大 碘单质的用量时(Entry 10), 未能分离得到目标产物 $5 \mathbf{a}$. 因此, 由化合物 4a 脱甲基形成化合物 5a 的最优化条件 为: 乙腈作为溶剂, 铝、碘和 $\mathbf{4 a}$ 的摩尔当量比为 3.6 : $5.4: 1$, 反应温度为 $75{ }^{\circ} \mathrm{C}$, 反应 $7 \mathrm{~h}$. 在最优化条件的基 础上, 对化合物 $4 \mathrm{~b}$ 和 $4 \mathrm{c}$ 进行脱甲基, 可以得到化合物 $\mathbf{5 b}$ (赤松素)和 $\mathbf{5 e}$ (白皮杉醇), 反应的收率分别为 $87 \%$ 和 68\% (Entries 12,13).

\section{2 脱甲基异构化反应机理研究}

以白蒜芦醇的合成为例, 化合物 $\mathbf{4 a}$ 在碘化铝的作
用下脱甲基的同时发生双键的顺反异构. 邹永等 ${ }^{[7]}$ 认为 双键异构的原因如下：一是 $\mathrm{AlI}_{3}$ 在脱甲基的同时，对双 键发生亲电加成再消除反应, 形成反式构型双键; 二是 高温可以促使双键发生由顺式到反式的构型转变.

在合成白莍芦醇时，首先以 $\mathrm{AlI}_{3}$ 作为脱甲基试剂， 但反应只得到 $1: 4$ 的顺反异构体混合物, 即使将反应 温度设置为 $82{ }^{\circ} \mathrm{C}$, 仍未能得到单一反式构型的白愁芦 醇. 结合已报道的单质碘和二苯基硫醚促进的双键顺反 异构研究 ${ }^{[8]}$, 推测出导致双键翻转的原因可能是由于碘 的存在, 碘在加热下形成的碘自由基. 碘自由基与双键 的作用可能通过两种途径, 如 Scheme 3 所示. 论文主要 讨论路径 $\mathrm{A}$, 碘自由基进攻双键，形成的新自由基中间 体 7, 在 $\mathrm{AlI}_{3}$ 的作用下完成甲基的脱除形成中间体 8 , 再 通过旋转形成更稳定的自由基中间体 $\mathbf{9}$, 反式消除得到 中间体 10, 水解得到反式构型的白藜芦醇 (path A). 另 外一种路径 $\mathrm{B}$, 则主要通过碘自由基与双键形成 $\pi$ 络合 物，再通过脱甲基、构型旋转和碘自由基脱除得到中间 体 10, 最后形成反式构型的白僽芦醇(path B). 而在 $\mathrm{AlI}_{3}$ 脱甲基过程中发生的双键顺反异构, 则可能是由于碘负 离子被氧化为碘单质，后者在加热下形成的碘自由基促 进双键顺反异构.

为了进一步验证反应机理的合理性, 我们进行了自 由基捕获实验的研究和理论计算. 如 Scheme 4 所示, 我 们首先以以化合物 $4 \mathrm{a}$ 为原料，向反应体系中加入 $10 \%$ 碘，室温反应 $30 \mathrm{~min}$, 可以得到全反式的 $\mathbf{4 a}$ (条件 c). 而 当在反应体系中加入 1 equiv.的 Tempo 时(条件 $\mathrm{d}$ ), 顺反 异构被完全抑制, 实验结果表明在碘促进的双键顺反异 构中, 碘自由基可能是关键因素. 在此基础上以化合物 


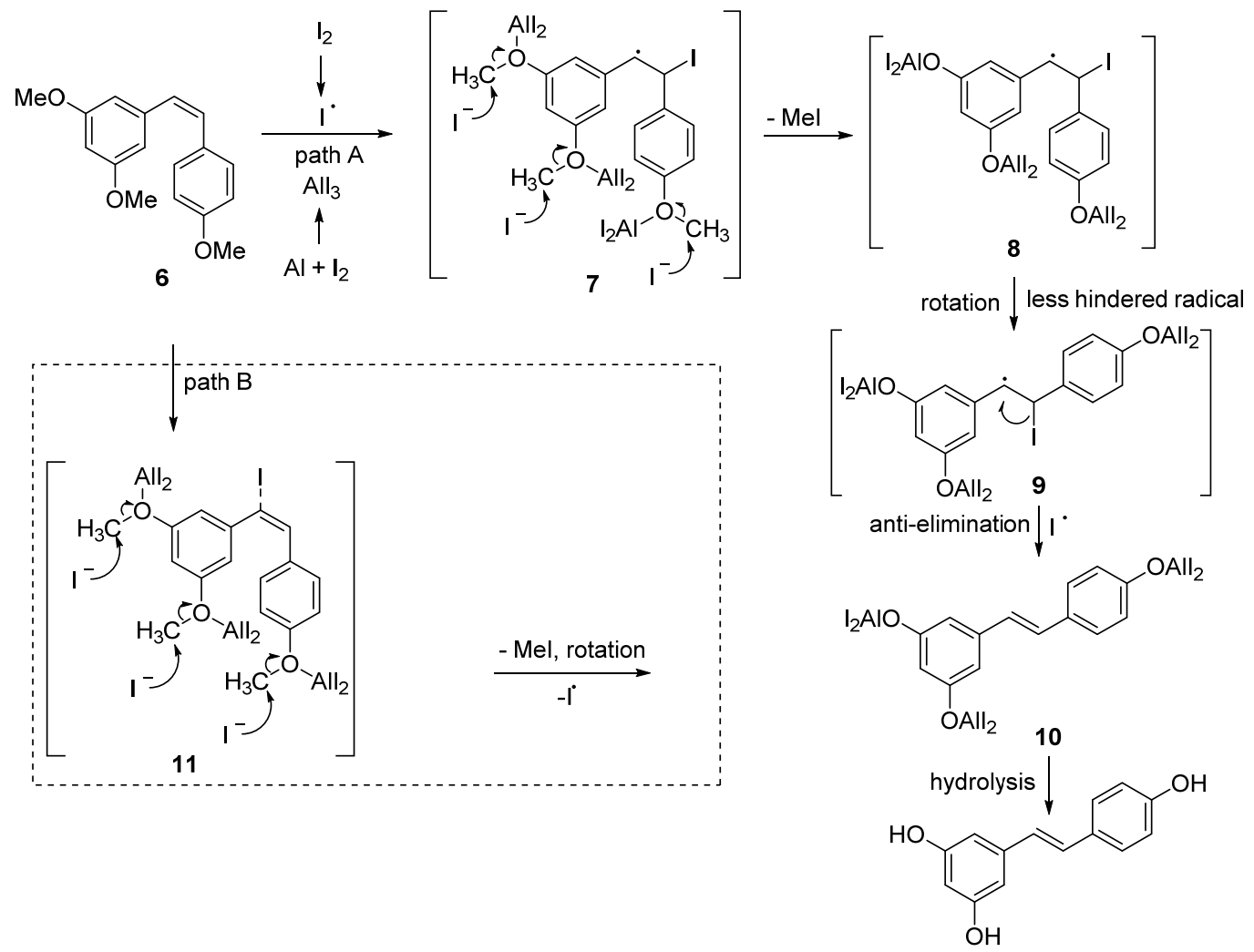

图式 3 脱甲基异构化反应机理

Scheme 3 Proposed mechanism for demethylation/isomerisation process

4a 为原料, 在反应体系中加入自由基捕获剂时, 反应的 收率和目标产物的顺反异构体的比例有所改变. 当加入 2,2,6,6-四甲基哌啶氧化物 (Tempo)时, 反应的收率为 $82 \%$, 但是目标产物 $5 \mathbf{a}$ 为 $1: 4$ 的顺反异构体混合物; 当加入 2,6-二叔丁基对甲酚(BHT)时, 产物 $\mathbf{5 a}$ 为单一反 式构型产物, 反应的收率为 $70 \%$. 加入 BHT 时, 酚可能 与脱甲基试剂中的铝盐反应的速度更快, 导致其无法起 到捕获自由基的作用. 加入 Tempo 可以影响目标产物 的异构体比例, 证实自由基在双键顺反中发挥关键作 用, 进一步将 Tempo 的用量增加至 6 或 10 equiv. 时, 脱 甲基反应被完全抑制，未能得到目标产物 $\mathbf{5 a}$.

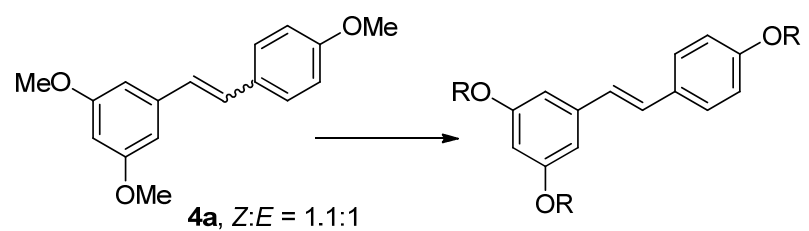

(a) 3.6 equiv. $\mathrm{Al}, 5.4$ equiv. $\mathrm{I}_{2}, \mathrm{CH}_{3} \mathrm{CN}, 75^{\circ} \mathrm{C}$ 1.0 equiv. Tempo.

(b) 3.6 equiv. Al, 5.4 equiv. $\mathrm{I}_{2}, \mathrm{CH}_{3} \mathrm{CN}, 75^{\circ} \mathrm{C}$ 1.0 equiv. $\mathrm{BHT}$

(c) $10 \% \mathrm{I}_{2}, \mathrm{CH}_{3} \mathrm{CN}$, r.t., $30 \mathrm{~min}$

(d) $10 \% \mathrm{I}_{2}, 1.0$ equiv. Tempo, $\mathrm{CH}_{3} \mathrm{CN}$, r.t.

$82 \%, Z: E=1: 5,5 a$

$70 \%$, only $E$, 5 a

E only, 4a

$Z: E=1.1: 1,4 a$

理论计算中, 使用 Gaussian 16 (Revision B.01)软件 对上述两种反应机理在 $\omega \mathrm{B} 97 \mathrm{XD} / \mathrm{def} 2-\mathrm{SVP} / \mathrm{IEFPCM}$ 水 平上进行了初步的量子化学计算, 对脱甲基异构化反应 机理中的路径 $\mathrm{A}$ 进行计算. 计算结果表明, $\mathrm{AlI}_{3}$ 与双键 的相互作用较弱, 不足以使 $\pi$ 键断裂发生亲电加成, 因 此反应通过邹永等人提出的机理 ${ }^{[7]}$ 进行的可能性较低, 而本论文中的双键异构步骤从吉布斯自由能变来看是 更合理的, 如图 2 所示, 相关计算结果见辅助材料.

\section{2 结论}

以 3,5-二甲氧基苄醇为原料合成磷叶立德 3 , 与苯 甲醛、对甲氧基苯甲醛和 3,4-二甲氧基苯甲醛反应得到 甲基保护的多羟基二苯乙烯 $(\mathbf{4 a} \sim 4 \mathrm{c})$. 化合物 $4 \mathrm{a} \sim 4 \mathrm{c}$ 在 铝和碘的作用下完成甲基的脱除和双键的顺反异构，实 现白㳟芦醇、白皮杉醇和赤松素的合成. 该合成方法可 以一锅反应实现双键的顺反异构和苯甲醚的甲基脱除, 也避免了三碘化铝的现制现用，反应操作更加简单，同 时将反应溶剂过滤后得到的滤液可以再作为脱甲基反 应的溶剂使用. 通过理论计算和自由基捕获实验，本文 认为在铝和碘促进的脱甲基异构化反应过程中, 碘自由 基可能是导致双键顺反异构的关键因素.

图式 4 控制实验

Scheme 4 Control experiments 


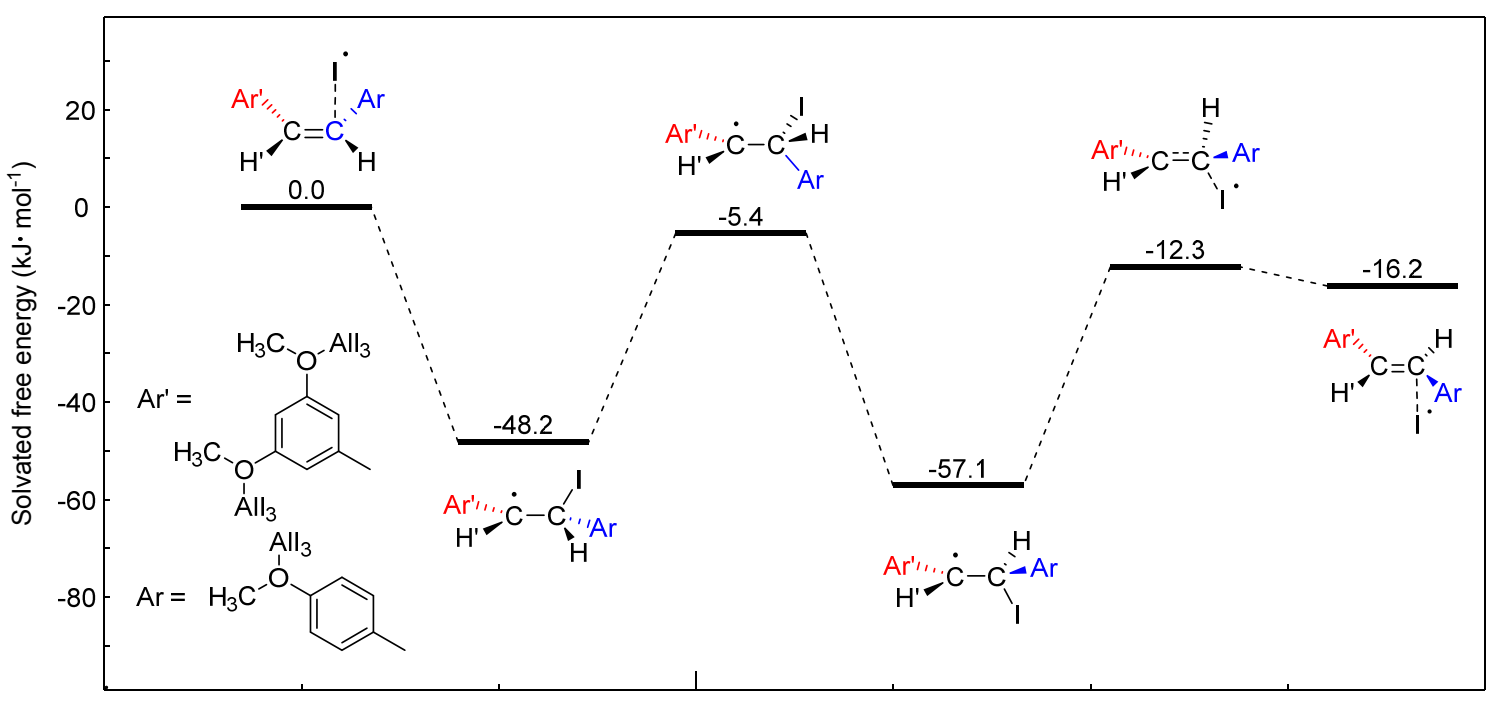

图 2 双键顺反异构化历程的自由能示意图

Figure 2 Free energy profile for the cis-trans isomerization of double bond

\section{3 实验部分}

\section{1 仪器与试剂}

核磁共振波谱仪: Bruker Avance $400 \mathrm{MHz}$ 型核磁共 振仪; 熔点仪: YRT-3 型熔点仪; 柱层析硅胶: $200 \sim 300$ 目 (青岛海洋). 除乙腈为 HPLC 级溶剂(含水量 $\leqslant 0.01 \%$ ), 其它实验所用试剂和原料均为分析纯. 势垒计算计算软 件采用 Gaussian 16, Revision B.01, 势能面扫描的计算 方为 $\omega$ B97XD 泛函, def2-SVP 基组.优化单点结构和计 算吉布斯自由能变时, 加入了 IEFPCM 溶剂模型, 设定 溶剂为乙腈, 温度为 $350 \mathrm{~K}$.

\section{2 实验方法}

\section{2 .1 化合物 $\mathbf{4 a} \sim \mathbf{4 c}$ 的合成}

在 $100 \mathrm{~mL}$ 的圆底烧瓶中加入叶立德 $3(12 \mathrm{mmol})$ 和 $40 \mathrm{~mL}$ 异丙醇, 搅拌下加入氢氧化锂 $(16 \mathrm{mmol})$, 加完 后反应 $15 \mathrm{~min}$, 再加入芳香醛 $(11 \mathrm{mmol})$, 加热至 $60{ }^{\circ} \mathrm{C}$ 反应至醛消耗完全 (TLC 监测). 加入 $40 \mathrm{~mL}$ 水淬灭反应, 用乙酸乙酯 $(80 \mathrm{~mL} \times 3)$ 萃取. 有机相先用饱和氯化钠洗, 再用无水硫酸钠干燥, 过滤, 减压除去溶剂, 得到油状 物, 硅胶柱层析分离 [洗脱剂: $V$ (乙酸乙酯) : $V$ (石油 醚) $=1: 20]^{[9]}$.

1,3-二甲氧基 -5-(4-甲氧基苯乙烯基) 苯 (4a) ${ }^{[9]}$ : $2.498 \mathrm{~g}$, 产率 $84 \% . Z: E=1.1: 1$, 无色油状物. ${ }^{1} \mathrm{H}$ NMR $\left(\mathrm{CDCl}_{3}, 400 \mathrm{M} \mathrm{Hz}\right) \delta: 7.45$ (d, $\left.J=8.8 \mathrm{~Hz}, 2 \mathrm{H}\right), 7.22$ $(\mathrm{d}, J=8.8 \mathrm{~Hz}, 2 \mathrm{H}), 7.05$ (d, $J=16 \mathrm{~Hz}, 1 \mathrm{H}), 6.929 \sim 6.891$ $(\mathrm{m}, 3 \mathrm{H}), 6.77(\mathrm{~d}, J=8.8 \mathrm{~Hz}, 2 \mathrm{H}), 6.65(\mathrm{~d}$, $J=2.4 \mathrm{~Hz}, 2 \mathrm{H}), 6.53(\mathrm{~d}, J=12 \mathrm{~Hz}, 1 \mathrm{H}), 6.462 \sim 6.437(\mathrm{~m}$, $3 \mathrm{H}), 6.38(\mathrm{t}, J=6.4 \mathrm{~Hz}, 1 \mathrm{H}), 6.346 \sim 6.285(\mathrm{~m}, 2 \mathrm{H}), 3.83$ (s, 6H), $3.78(\mathrm{~s}, 3 \mathrm{H}), 3.78(\mathrm{~s}, 3 \mathrm{H}), 3.67(\mathrm{~s}, 6 \mathrm{H})$.

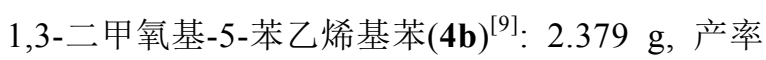
$90 \% . Z: E=2: 1.7$, 无色油状物. ${ }^{1} \mathrm{H}$ NMR $\left(\mathrm{CDCl}_{3}, 400\right.$ $\mathrm{M} \mathrm{Hz}) \delta: 7.522 \sim 7.503(\mathrm{~m}, 2 \mathrm{H}), 7.380 \sim 7.342(\mathrm{~m}, 2 \mathrm{H})$, $7.288 \sim 7.186(\mathrm{~m}, 6 \mathrm{H}), 7.12(\mathrm{dd}, J=16 \mathrm{~Hz}, 2 \mathrm{H}), 6.68(\mathrm{~d}$, $J=2.4 \mathrm{~Hz}, 2 \mathrm{H}), 6.62(\mathrm{~d}, J=12.4 \mathrm{~Hz}, 1 \mathrm{H}), 6.53$ (d, $J=12.4$ $\mathrm{Hz}, 1 \mathrm{H}), 6.411 \sim 6.399(\mathrm{~m}, 3 \mathrm{H}), 6.344 \sim 6.290(\mathrm{~m}, 3 \mathrm{H})$, 3.84 (s, 3H), 3.77 (s, 3H), 3.64 (s, 6H).

4-(3,5-二甲氧基苯乙烯基)-1,2-二甲氧基苯(4c) ${ }^{[9]}$ : $2.709 \mathrm{~g}$, 产率 $82 \% . Z: E=1.1: 1$, 无色油状物. ${ }^{1} \mathrm{H}$ NMR $\left(\mathrm{CDCl}_{3}, 400 \mathrm{M} \mathrm{Hz}\right) \delta: 7.056 \sim 7.015(\mathrm{~m}, 3 \mathrm{H}), 6.91$ $(\mathrm{d}, J=16.4 \mathrm{~Hz}, 1 \mathrm{H}), 6.871 \sim 6.832(\mathrm{~m}, 3 \mathrm{H}), 6.75(\mathrm{~d}, J=$ $8.4 \mathrm{~Hz}, 1 \mathrm{H}), 6.66(\mathrm{~d}, J=2.4 \mathrm{~Hz}, 2 \mathrm{H}), 6.52(\mathrm{~d}, J=12.4 \mathrm{~Hz}$, $1 \mathrm{H}), 6.486 \sim 6.456(\mathrm{~m}, 3 \mathrm{H}), 6.38(\mathrm{t}, J=6.4 \mathrm{~Hz}, 1 \mathrm{H}), 6.32$ $(\mathrm{t}, J=6.3 \mathrm{~Hz}, 1 \mathrm{H}), 3.95(\mathrm{~s}, 3 \mathrm{H}), 3.91(\mathrm{~s}, 3 \mathrm{H}), 3.86(\mathrm{~s}, 3 \mathrm{H})$, $3.83(\mathrm{~s}, 6 \mathrm{H}), 3.68(\mathrm{~s}, 6 \mathrm{H}), 3.65(\mathrm{~s}, 3 \mathrm{H})$.

\section{2 .2 化合物 $\mathbf{5 a} \sim \mathbf{5} \mathbf{c}$ 的合成}

在 $250 \mathrm{~mL}$ 圆底烧瓶中, 零度下依次向反应瓶中加 入乙腈 $(150 \mathrm{~mL})$, 铝 $(15 \mathrm{mmol})$, 碘 $(21.6 \mathrm{mmol})$ 和化合物 4a 4c $(4 \mathrm{mmol})$, 加完后升温至 $75{ }^{\circ} \mathrm{C}$ 反应 $7 \mathrm{~h}$, 冷却至 室温. 将反应烧瓶置于冰浴下, 加入 $20 \mathrm{~mL} 2 \mathrm{~mol} / \mathrm{L} \mathrm{HCl}$ 溶液, 搅拌 $0.5 \mathrm{~h}$, 过滤除去铝粉, 乙酸乙酯 $(50 \mathrm{~mL} \times 3)$ 萃取, 有机相用饱和氯化钠洗涤, 无水硫酸钠干燥, 过 滤, 减压除去溶剂, 得到的粗产物用硅胶柱层析分离 [洗脱剂: $V$ (乙酸乙酯 $): V$ (石油醚 $)=1.50: 1.26$ $260]^{[10]}$.

白藜芦醇(5a): $739.5 \mathrm{mg}$, 产率 $81 \%$. 白色固体, m.p. $257 \sim 259{ }^{\circ} \mathrm{C} \quad$ (lit. ${ }^{[10 b]}$ m.p. 259 $260{ }^{\circ} \mathrm{C}$ ); ${ }^{1} \mathrm{H}$ NMR (DMSO-d $\left.{ }_{6}, 400 \mathrm{MHz}\right) \delta: 9.58(\mathrm{~s}, 1 \mathrm{H}), 9.23(\mathrm{~s}, 2 \mathrm{H}), 7.40$ $(\mathrm{m}, 2 \mathrm{H}), 6.94(\mathrm{~d}, J=16 \mathrm{~Hz}, 1 \mathrm{H}), 6.82(\mathrm{~d}, J=16 \mathrm{~Hz}, 1 \mathrm{H})$, 
$6.76(\mathrm{~m}, 2 \mathrm{H}), 6.40(\mathrm{~d}, J=2.4 \mathrm{~Hz}, 2 \mathrm{H}), 6.14(\mathrm{t}, J=2.0 \mathrm{~Hz}$, $1 \mathrm{H}) ;{ }^{13} \mathrm{C}$ NMR (DMSO- $\left.d_{6}, 100 \mathrm{M} \mathrm{Hz}\right) \delta$ : 159.0, 157.7, 139.7, 128.5, 128.3, 128.3, 126.1, 116.0, 104.8, 102.2.

赤松素(5b): $738.6 \mathrm{mg}$, 产率 87\%. 黄色固体, m.p. $152 \sim 154{ }^{\circ} \mathrm{C}$ (lit. ${ }^{[10 b]}$ m.p. $\left.149 \sim 151{ }^{\circ} \mathrm{C}\right) ;{ }^{1} \mathrm{H}$ NMR $\left(\mathrm{DMSO}-d_{6}, 400 \mathrm{MHz}\right) \delta: 9.28(\mathrm{~s}, 2 \mathrm{H}), 7.58(\mathrm{~d}, J=6.8 \mathrm{~Hz}$, $2 \mathrm{H}), 7.38 \sim 7.34(\mathrm{~m}, 1 \mathrm{H}), 7.27 \sim 7.24(\mathrm{~m}, 2 \mathrm{H}), 7.09 \sim$ $7.01(\mathrm{~m}, 2 \mathrm{H}), 6.45(\mathrm{~d}, J=2.0 \mathrm{~Hz}, 2 \mathrm{H}), 6.17$ (t, $J=2.0 \mathrm{~Hz}$, $1 \mathrm{H}) ;{ }^{13} \mathrm{C}$ NMR (DMSO- $\left.d_{6}, 100 \mathrm{MHz}\right) \delta: 159.0,139.2$, $137.5,129.4,129.1,128.3,128.0,126.9,105.2,102.8 .{ }^{1} \mathrm{H}$ NMR (400 M Hz, acetone- $\left.d_{6}\right) \delta: 8.26(\mathrm{~s}, 2 \mathrm{H}), 7.60 \sim 7.58$ $(\mathrm{m}, 2 \mathrm{H}), 7.39 \sim 7.35(\mathrm{~m}, 2 \mathrm{H}) 7.27(\mathrm{~m}, 1 \mathrm{H}), 7.12(\mathrm{dd}, J=$ $16.8,16.8 \mathrm{~Hz}, 2 \mathrm{H}), 6.61(\mathrm{~d}, J=2.2 \mathrm{~Hz}, 2 \mathrm{H}), 6.33(\mathrm{t}, J=2.2$ $\mathrm{Hz}, 1 \mathrm{H}) ;{ }^{13} \mathrm{C}$ NMR $\left(100 \mathrm{M} \mathrm{Hz}\right.$, acetone- $\left.d_{6}\right) \delta: 158.7,139.5$, $137.5,128.9,128.6,128.3,127.5,126.5,105.2,102.3$.

白皮杉醇(5c): $664.3 \mathrm{mg}$, 产率 68\%. 白色固体, m.p. $233 \sim 235{ }^{\circ} \mathrm{C} \quad\left(\right.$ lit. ${ }^{[10 a]}$ m.p. $\left.231 \sim 234{ }^{\circ} \mathrm{C}\right) ;{ }^{1} \mathrm{H}$ NMR $\left(\mathrm{DMSO}-d_{6}, 400 \mathrm{MHz}\right) \delta: 9.20 \sim 8.97(\mathrm{~m}, 4 \mathrm{H}), 6.95(\mathrm{~d}, J=$ $2.4 \mathrm{~Hz}, 2 \mathrm{H}), 6.84(\mathrm{~d}, J=16.0 \mathrm{~Hz}, 1 \mathrm{H}), 6.83(\mathrm{dd}, J=8.0$, $2.0 \mathrm{~Hz}, 1 \mathrm{H}), 6.70(\mathrm{~d}, J=8.0 \mathrm{~Hz}, 1 \mathrm{H}), 6.71(\mathrm{~d}, J=16.0 \mathrm{~Hz}$, $1 \mathrm{H}), 6.36(\mathrm{~d}, J=2.4 \mathrm{~Hz}, 2 \mathrm{H}), 6.10(\mathrm{t}, J=2.2 \mathrm{~Hz}, 1 \mathrm{H}) ;{ }^{13} \mathrm{C}$ NMR (DMSO- $\left.d_{6}, 100 \mathrm{MHz}\right) \delta: 158.6,145.6,145.5,139.4$, 128.7, 128.4, 125.6, 118.7, 115.8, 113.4, 104.4, 101.8.

辅助材料(Supporting Information) 化合物 $4 \mathrm{a} \sim \mathbf{4 c}$ 和 5a 5c 的核磁共振谱图; 反应机理的量子化计算结果. 这些材料可以免费从本刊网站(http://sioc-journal.cn/)上 下载.

\section{References}

[1] (a) Filippis, B. D.; Ammazzalorso, A.; Amoroso, R.; Giampietro, L. Drug Dev. Res. 2019, 80, 285.

(b) Keylor, M. H.; Matsuura, B. S.; Stephenson, C. R. Chem. Rev. 2015, 115, 8976.

(c) Si, J.-Y. Nat. Prod. Res. Dev. 1994, 6, 71 (in Chinese). (斯建勇, 天然产物研究与开发, 1994, 6, 71.)

[2] Chen, G. H.; Webster, J. M.; Li, J. X.; Hu, K. J.; Zhu, J. WO 042231, 2001 [Chem. Abstr. 2001, 135, 45986.]

[3] (a) Quideau, S.; Deffieux, D.; Pouysegu, L. Angew. Chem., Int. Ed. 2012, 51,6824 .

(b) Gao, H.; Zheng, X.; Qi, Y.; Wang S.; Wan C.; Rao, G.; Mao, Z. Chin. J. Org. Chem. 2018, 38, 648 (in Chinese).

(高慧, 郑喜, 祁燕, 王斯, 万春平, 饶高雄, 毛泽伟, 有机化学, 2018, 38, 648.)

[4] (a) Becker, K. B. Synthesis 1983, 341.

(b) Zhao, S.; Yu, Y.; Zhang, Y. Chin. J. Org. Chem. 2013, 33, 1851.

(c) Khan, Z. A.; Iqbal, A.; Shahzad, S. A. Mol. Diversity 2017, 21, 483.

[5] (a) Li, Q.; Shah, Z.; Qu, J. Kang, Y. J. Org. Chem. 2018, 83, 296. (b) Srivastava, V. Catal. Lett. 2017, 147, 693.

(c) Zou, Y.; Huang, Q.; Huang, T.; Ni, Q.; Zhang, E.; Xu, T.; Yuan, M.; Li, J. Org. Biomol. Chem. 2013, 11, 6867.

(d) Gray, E. E.; Rabenold, L. E.; Goess, B. C. Tetrahedron Lett. 2011, 52, 6177.

(e) Sun, H.-Y.; Xiao, C.-F.; Cai, Y.-C.; Chen, Y.; Wei, W.; Liu, X.-K.; Lv, Z.-L.; Zou, Y. Chem. Pharm. Bull. 2010, 58, 1492.

(f) Alonso, F.; Riente, P.; Yus, M. Eur. J. Org. Chem. 2009, 34, 6034.

(g) Dong, D. J.; Li, H. H.; Tian, S. K. J. Am. Chem. Soc. 2010, 132, 5018 .

(h) Alonso, F.; Riente, P.; Yus, M. Tetrahedron Lett. 2009, 50, 3070.

(i) McNulty, J.; Das, P. Eur. J. Org. Chem. 2009, 24, 4031.

(j) Roberti, M.; Pizzirani, D.; Simoni, D.; Rondanin, R.; Baruchello, R.; Bonora, C.; Buscemi, F.; Grimaudo, S.; Tolomeo, M. J. Med. Chem. 2003, 46, 3546.

(k) Ali, M. A.; Kondo, K.; Tsuda, Y. Chem. Pharm. Bull. 1992, 40, 1130.

[6] (a) Tian, J.; Yue, H.; Yang, P.; Sang, D. ChemistrySelect 2019, 4, 38.

(b) Sang, D.; Tian, J.; Tu, X.; He, Z.; Yao, M. Synthesis 2019, 51, 704.

(c) Sang, D.; Tu, X.; Tian, J.; He, Z.; Yao, M. ChemistrySelect 2018, 3, 10103.

(d) Sang, D.; Yao, M.; Tian, J.; Chen, X.; Zhan, H.; You, L. Synlett 2017, 28, 138

[7] (a) Li, G.; Zou, Y.; Zhang, X. J. Chem. Res. 2007, 657.

(b) Sun, H.; Xiao, C.; Wei, W.; Chen, Y.; Lu, Z.; Zou, Y. Chin. J. Org. Chem. 2010, 30, 1574 (in Chinese).

(孙洪宜, 肖春芬, 魏文, 陈显, 吕泽良, 邹永, 有机化学, 2010, 30,1574 .)

[8] (a) van Rossum, A. J. G.; de Bruin, A. H. M.; Nivard, R. J. F. J. Chem. Soc., Perkin Trans. 2 1975, 1036.

(b) Giacomelli, G.; Lardicci, L.; Saba, A. J. Chem. Soc., Perkin Trans. 1 1978, 314.

(c) Ali, M. A.; Tsuda, Y. Chem. Pharm. Bull. 1992, 40, 2842.

(d) Gaukroger, K.; Hadfield, J. A.; Hepworth, L. A.; Lawrence, N. J.; McGown, A. T. J. Org. Chem. 2001, 66, 8135.

(e) Hepprele, S. S.; Li, Q.; East, A. L. L. J. Phys. Chem. A 2005, 109, 10975.

(f) Deshpande, P. B.; Kumar, S.; Palanisamy, U.; Andrew, G. US $6844471,2005$.

(g) Li, Q.; Shah, Z.; Qu, J.; Kang, Y. J. Org. Chem. 2018, 83, 296.

(h) Settle, A. E.; Berstis, L.; Zhang, S.; Rorrer, N. A.; Hu, H.; Richards, R. M.; Beckham, G. T.; Crowley, M. F.; Vardon, D. R. ChemSusChem 2018, 11, 1768.

[9] (a) Das, M.; O'Shea, D. F. Org. Lett. 2016, 18, 336.

(b) Simoni, D.; Roberti, M.; Invidiata, F. P.; Aiello, E.; Aiello, S.; Marchetti, P.; Baruchello, R.; Eleopra, M.; Cristina, A. D.; Grimaudo, S.; Gebbia, N.; Crosta, L.; Dieli, F.; Tolomeo, M. Bioorg. Med. Chem. Lett. 2016, 16, 3245.

(c) Zaki, M. A.; Balachandran, P.; Khan, S.; Wang, M.; Mohammed, R.; Hetta, M. H.; Pasco, D. S.; Muhammad, I. J. Nat. Prod. 2013, 76, 679 .

(d) Antonioletti, R.; Bonadies, F.; Ciammaichella, A.; Viglianti, A. Tetrahedron 2008, 64, 4644.

[10] (a) Sun, H.-Y.; Xiao, C.-F.; Cai, Y.-C.; Chen, Y.; Wei, W.; Liu, X.-K.; Lv, Z.-L.; Zou, Y. Chem. Pharm. Bull. 2010, 58, 1492.

(b) Jayatilake, G. S.; Jayasuriya, H.; Lee, E. S.; Koonchanok, N. M.; Geahlen, R. L.; Ashendel, C. L.; McLaughlin, J. L.; Chang, C. J. J. Nat. Prod. 1993, 56, 1805.

(c) Uzura, S.; Sekine-Suzuki, E.; Nakanishi, I.; Sonoda, M.; Tanimori, S. Bioorg. Med. Chem. Lett. 2016, 26, 3886. 